\title{
Análise da descentralização no desenvolvimento de novos produtos: uma investigação em uma empresa do setor de veículos comerciais
}

\author{
André Segismundo ${ }^{\mathrm{a} *}$, Paulo Augusto Cauchick Miguel ${ }^{\mathrm{b}}$ \\ a*segismundo_andre@yahoo.com, USP, Brasil \\ bcauchick@usp.br, USP, Brasil
}

\begin{abstract}
Resumo
A literatura atual identifica uma tendência de descentralização no desenvolvimento de novos produtos em âmbito global. Nesse contexto, o objetivo do presente trabalho é analisar a descentralização no desenvolvimento global de produtos (DGP) em uma montadora automotiva. Os fatores que favorecem a descentralização do DGP são considerados como variáveis na investigação, bem como as suas causas. Dada a natureza dos dados (qualitativos), a relevância do contexto (setor automotivo) e o propósito da pesquisa, a abordagem metodológica de estudo de caso é adotada, conduzida em uma montadora de veículos comerciais na unidade no Brasil e na matriz no exterior. Os resultados indicam que a organização investigada reage de maneira similar a outros setores econômicos (como, por exemplo, o setor de telecomunicações), quando se depara com a decisão de descentralização do DGP. 0 interesse no mercado local e aspectos históricos da evolução da subsidiária brasileira mostraram-se relevantes. Identificou-se também que o produto modular influencia no processo de descentralização do DGP, contraditoriamente a estudos anteriores. A partir desses resultados, é então feita uma proposta de associação entre o tipo de estrutura de DGP e a utilização de projeto modular.
\end{abstract}

Palavras-chave

Indústria automobilística. Descentralização no desenvolvimento de produto. Modularidade.

\section{Introdução}

Nas últimas décadas, o desenvolvimento de novos produtos (DP) vem se tornando um processo de negócio cada vez mais crítico para a competitividade das empresas. Dentre as diversas razões estão a crescente internacionalização econômica, aumento da diversidade e variedade nas ofertas de produtos, bem como pela redução do ciclo de vida dos produtos no mercado (OPPAT, 2008). É nesse ambiente de crescente complexidade e competitividade global e de importância estratégica do desenvolvimento de produto (DP) para as empresas e suas estratégias competitivas que organizações transnacionais (transnational companies - TNCs) têm intensificado o processo de descentralização de suas estruturas de desenvolvimento de produtos (MUDAMBI; MUDAMBI; NAVARRA, 2007).
No Brasil, nos últimos anos diversos produtos foram desenvolvidos com intensiva participação da engenharia das subsidiárias brasileiras das montadoras automotivas (QUINTELLA; ROCHA, 2007). Exemplos de sucesso de mercado incluem os modelos Celta e Meriva (GM), Pálio (parcialmente desenvolvido sob a responsabilidade da engenharia da Fiat Brasil), Polo Sedan (para mercados como Brasil, China e África do Sul) e o Fox da Volkswagen, este último, inclusive, exportado para a Europa. Outros estudos (CHIESA, 2000; SUBRAMANIAM, 2006; HARVEY; GRIFFITH, 2007) mostram ainda que o desenvolvimento de novos produtos em muitas TNCs apresenta um caráter cada vez mais descentralizado, com times de engenharia distribuídos entre matriz e filiais muitas vezes trabalhando conjuntamente em projetos de 
novos veículos. No entanto, em geral esses estudos consideram montadoras cujos produtos são automóveis diferentemente da análise de veículos comerciais, foco do presente trabalho.

Considerando-se a complexidade do desenvolvimento de novos produtos, destacada na literatura (e.g. em ROZENFELD et al., 2006), sobretudo neste âmbito internacional, o contexto de descentralização de negócios e do desenvolvimento global de produtos (DGP) - conceito apresentado a seguir - e, ainda, a importância de estudos sobre a indústria automobilística no âmbito nacional e internacional, o presente trabalho tem como objetivo analisar a descentralização no desenvolvimento de novos produtos adotada por uma empresa automotiva, enfatizando as relações entre a sua a matriz e a subsidiária brasileira atuante no desenvolvimento e produção de veículos comerciais.

\section{Referencial teórico - desenvolvimento de produto, tipos de projetos e desenvolvimento global de produtos}

0 desenvolvimento de produto (DP) pode ser definido como um dos processos de maior intensidade de concentração de conhecimento nos negócios de uma organização e, por si só, está constantemente gerando novos conhecimentos (SÖDERQUIST, 2006). Alguns fatores relevantes para a tomada de decisão quanto à distribuição dessas atividades complexas de DP pelas TNCs ao redor do globo são discutidos na literatura e apresentados a seguir.

\subsection{Classificação dos projetos de desenvolvimento de produto e tipos de arquitetura}

Os principais tipos de projetos de DP com relação à amplitude na alteração das especificações do produto ou do processo de produção são classificados, segundo Clark e Wheelwright (1993): projetos incrementais e derivativos; próxima geração ou plataforma; inovações radicais; pesquisa e desenvolvimento avançado; e projetos de parceria (alianças). Essa classificação considera uma categorização dos projetos de acordo com seu grau de inovação, partindo daqueles com menor grau de inovação associada, por exemplo, a pequenas melhorias de um produto já existente até aqueles projetos de pesquisa avançada, que trazem uma inovação significativa, resultando em soluções inexistentes até então. A definição do tipo de projeto é um dos fatores importantes na decisão de descentralização ou não do DGP, como destacado mais à frente.
Outra importante classificação considera a estrutura de um produto, que representa o esquema no qual os elementos funcionais do produto são arranjados em conjuntos e subconjuntos e como estes interagem entre si (MUFFATTO, 1999; HUANG, 2000). Nesse sentido, a estrutura do produto pode ser classificada em "integrada" ou "modular" (ULRICH; EPPINGER, 1995). A primeira categoria apresenta como as principais características dos elementos funcionais do produto são implementadas usando mais de um bloco, ou seja, um subconjunto de componentes substituíveis que realizam funções similares (HUANG, 2000). Cada um desses blocos isolados deve considerar vários elementos funcionais e, em geral, as interações entre os blocos não são bem definidas e devem apresentar uma relação causal à função primária do produto. 0 produto modular é derivado da abordagem de se organizar produtos ou processos complexos de uma forma mais eficiente (BALDWIN; CLARK, 1997), por meio da decomposição destes em subconjuntos mais simples que podem ser gerenciados de forma independente (MIKKOLA, 2001).

No que tange ao conceito de modularidade, mais especificamente associado ao desenvolvimento de produto, este pode ser definido como a escolha das fronteiras de projeto de um produto e seus componentes, como, por exemplo, na divisão de um sistema em módulos, de maneira que as funções e tarefas sejam interdependentes dentro do módulo e independentes entre os diferentes módulos que compõem o produto (HUANG; KUSIAK, 1998; CAMUFFO, 2001; PANDREMENOS et al., 2009). Nesse sentido, um dos principais aspectos da modularidade é a sua influência na estrutura do produto, o que, consequentemente, afeta o seu desenvolvimento. Uma das importantes tarefas no DP é identificar módulos comuns entre os produtos de maneira a possibilitar a otimização de plataformas de produtos, gerando-se assim uma família de produtos ou, ainda, identificar módulos comuns para serem desenvolvidos em parceria entre empresas na cadeia de fornecimento automotiva ou diferentes unidades de DP de uma mesma empresa (HÖLTTA; TANG; SEERING, 2003). Na realidade, o desenvolvimento de um novo produto, por diferentes unidades da mesma organização, pode ocorrer em nível global, como descrito a seguir.

\section{2. $O$ conceito de $D G P$}

0 conceito de "desenvolvimento global de produtos" (DGP) se difere do conceito de "desenvolvimento de produto global". No primeiro conceito, o termo "global" se refere à estrutura organizacional para o desenvolvimento de produto de uma organização transnacional, com unidades 
distribuídas em diferentes países que interagem entre si de diferentes maneiras, cujo resultado de um trabalho conjunto pode ser, por exemplo, aplicado em apenas um mercado específico (CHIESA, 1995, 1999). No caso de desenvolvimento de produto global, o termo "global" está associado ao produto e não à estrutura organizacional para o seu desenvolvimento, remetendo-se ao conceito dos anos 90 de desenvolver uma "plataforma mundial" de produto (HUMPHREY; LECLER; SALERNO, 2001; DIAS, 2003).

Estudos anteriores mostraram que a indústria automobilística tendia a centralizar suas atividades de pesquisa e desenvolvimento de produtos em poucos países. Em geral, essa centralização ocorria naqueles paises onde as matrizes de suas empresas multinacionais estavam instaladas (MILLER, 1994; CALABRESE, 2001). Essa tendência é controversa em estudos subsequentes. Dias (2003) e Consoni (2004) argumentam que não há padrões claramente adotados como consenso entre as montadoras de veículos no que tange à centralização ou descentralização do desenvolvimento de produto. Tais decisões são baseadas na estratégia da empresa e ao seu "grau de controle hierárquico predominante", entendido aqui como o grau de autonomia que as montadoras atribuem ou não às suas subsidiárias. Este grau de autonomia varia entre os seguintes conceitos, segundo Bélis-Bergouignan, Bordenave e Lung (1996): "internacionalização", onde há expansão geográfica, porém a relação centro-periferia é assimétrica, e "globalização", onde valorizam-se as competências das diferentes regiões no globo no desenvolvimento de um novo produto.

No Brasil também existem trabalhos que abordam, direta ou indiretamente, o DGP (e.g. SALERNO et al., 2001; TOLEDO et al., 2003, 2008; SILVA et al., 2004). Salerno, Camargo e Lemos (2008) concluíram que, em geral, a divisão internacional do trabalho nas TNCs deixa no exterior as atividades mais nobres de projeto de um novo produto. Isso significa que as fases de concepção do produto e elaboração de desenhos de engenharia (projeto detalhado) são realizadas nas matrizes. A participação das subsidiárias brasileiras cresce à medida que se afasta das atividades dessas duas fases rumo às atividades de adaptação de produto ou do processo de produção às condições locais. Tal fato vem ao encontro da tendência de centralização de atividades de desenvolvimento de produto já levantada por Miller (1994) no passado e por Calabrese (2001), cujo "efeito colateral" pode ser apenas a delegação de atividades de desenvolvimento de produto de menor valor agregado às subsidiárias.

Em uma análise sobre a descentralização de DGP e o envolvimento das subsidiárias brasileiras em um setor industrial diferente do automotivo, o de telecomunicações, foram identificados na literatura (em GALINA; PLONSKI, 2000; GALINA, 2003) fatores que justificavam a implantação ou manutenção de atividades de desenvolvimento de produto localmente. Com base nesses fatores, descritos a seguir, Galina (2003) propõe uma relação de "causa x efeito" que retrata a influência na participação brasileira no DGP do setor de telecomunicações. Como "causas", foram listadas as características gerais de empresas que tenham um DGP frente a alguns fatores identificados como influentes na escolha do local para instalar as unidades de desenvolvimento de produto especialmente no caso das subsidiárias brasileiras de telecomunicações. A partir desses fatores é possível identificar o comportamento da empresa, ou seja, os efeitos que esta incorre e que levam a uma probabilidade alta ou baixa de participação no DGP, como destacado no Quadro 1.

Outros estudos sobre DGP nas unidades brasileiras da Fiat (e.g. TOLEDO et al., 2003), GM e VW mostram que, em todos os casos, a participação da engenharia brasileira no DP foi maior nas fases finais do projeto e/ou no desenvolvimento de projetos do tipo derivativos. As justificativas de tais constatações referem-se, sobretudo, às características das etapas do processo de desenvolvimento de produto (PDP), ou seja, para um projeto do tipo plataforma, existe maior relação com as estratégias da empresa no início do desenvolvimento do produto. No caso de projetos do tipo derivativos, busca-se uma conciliação de escala e escopo e sensibilidade ao mercado local e diminuição de tempos e custos de projeto. Esses fatores auxiliam a justificar a divisão entre projetos plataformas e derivativos (DIAS, 2003).

Ainda, nesses estudos, não se identificou em nenhum deles a divisão do produto em módulos passíveis de serem desenvolvidos independentemente,

Quadro 1. "Causa × efeito" na participação das subsidiárias no desenvolvimento de produto.

\begin{tabular}{|l|c|}
\hline \multicolumn{1}{|c|}{ Causa(s) } & Avaliação dos efeitos \\
\hline $\begin{array}{l}\text { Interesse no mercado brasileiro } \\
\text { e latino }\end{array}$ & Alto - P, Baixo - NP \\
\hline Competências locais & Existe - P, Não existe - NP \\
\hline $\begin{array}{l}\text { Custo de desenvolvimento } \\
\text { local }\end{array}$ & Alto - P, Baixo - NP \\
\hline Autonomia da subsidiária & Existe - P, Não existe - NP \\
\hline Incentivos fiscais & Existe - P, Não existe - NP \\
\hline Tipo de produto & $\begin{array}{c}\text { Estratégico localmente - P, Não } \\
\text { estrat. localmente - NP }\end{array}$ \\
\hline Aspectos históricos & $\begin{array}{r}\text { Favorável ao DP - P, Não } \\
\text { favorável ao DP - NP }\end{array}$ \\
\hline Exigências governamentais & $\begin{array}{r}\text { Exigências para adaptações - P, } \\
\text { Não exigência - NP }\end{array}$ \\
\hline
\end{tabular}

Efeitos para a subsidiária: *P = alta probabilidade de participar do DGP; ${ }^{* *} \mathrm{NP}$ = baixa probabilidade de participar no DGP. Fonte: Adaptado de Galina e Plonski (2000). 
por equipes de engenharia de unidades diferentes, localizadas em lugares diferentes dentro da corporação. Identificou-se, no entanto, a divisão do produto em alguns módulos que são desenvolvidos por fornecedores, isto é, por equipes diferentes pertencentes a empresas diferentes: montadora e fornecedores (ver DIAS, 2003; SALERNO, 2002).

Para subsidiar melhor essa fundamentação teórica, faz-se necessário apresentar os tipos de unidades de desenvolvimento de produto no exterior, como contexto para o DGP, apresentado na sequência.

\subsubsection{Tipos de unidades de desenvolvimento de produto no exterior}

A literatura propõe basicamente dois tipos de unidades de desenvolvimento de produto de empresas multinacionais no exterior. A primeira classificação é feita por Ronstadt (1977), mais tarde atualizada por Reddy (1997), e distingue cinco tipos de unidades de DP por tipo de atividades por estas desenvolvidas:

- TTUs (Technology Transfer Units) ou Unidades de Transferência Tecnológica (apenas para servir como apoio local para serviços e para as facilidades de produção);

- ITUs (Indigenous Technology Units) ou Unidades de Tecnologia Nativa (para desenvolver novos produtos para atender o mercado local, adaptados ou desenvolvidos com tecnologia local);

- GTUs (Global Technology Units) ou Unidades de Tecnologia Global (para desenvolver novos produtos e processos para mercados internacionais ou globais);

- CTUs (Corporate Technology Units) ou Unidades de Tecnologia Corporativa (destinadas à geração de tecnologia de base de longo prazo ou exploratória para uso da empresa matriz); e

- RTUs (Regional Technology Units) ou Unidades de Tecnologia Regional.

Este quinto tipo de classificação, desenvolvido em mais detalhes por Reddy e Sigurdson (1994), refere-se a unidades destinadas a desenvolver produtos $\mathrm{e}$ processos em mercados regionais, sendo resultado da evolução da dinâmica da competitividade mais recente e tendências crescentes de descentralização do DP.

Outra classificação, estabelecida por Bartlett e Ghoshal (1989) e mais tarde discutida em sucessivas publicações por Chiesa $(1995,1996,1999)$, considera as unidades de desenvolvimento de produto no exterior em três tipos principais: $P \& D$ de adaptação; $P \& D$ originalmente local, destinado ao desenvolvimento de produtos originais para o atendimento do mercado local, e P\&D originalmente global, sendo uma unidade com papel especializado num processo de $\mathrm{P} \& \mathrm{D}$ coordenado centralmente.

Um trabalho realizado na Índia para identificar tendências de descentralização em DP naquele país concluiu que, num período de quase três décadas, a participação do país em termos de unidades de desenvolvimento de produto com maior grau de importância ou liberdade nas atividades aumentou consideravelmente (REDDY, 1997). Ainda segundo o autor supracitado, no final da década de 1990, das 32 empresas multinacionais analisadas com unidades de desenvolvimento de produto na Índia, quase a metade já era do tipo RTU, GTU e, inclusive, CTU. Tal fato ilustra o crescimento do país em termos de importância em participação em DGP (REDDY, 1997).

Estudos de natureza similar no Brasil, realizados na década de 1990 por Fleury e Fleury (2000), identificaram três modos de integração das subsidiárias nas matrizes: as subsidiárias podem atuar como um "braço operacional" das organizações transacionais; como uma "unidade relativamente autônoma"; ou como um "centro de competências". Em cada situação, a forma de relacionamento entre as unidades matriz e subsidiária é diferente, sendo a primeira situação a mais centralizadora em termos de processos decisórios, e, em termos de desenvolvimento de produto, apresenta sobretudo alta padronização no âmbito global e uma baixa customização local. 0 Quadro 2 sintetiza as principais características das três abordagens quanto ao processo decisório e projeto do produto, propostas por Fleury e Fleury (2000).

Quadro 2. Características das diferentes abordagens das corporações com relação às suas subsidiárias.

\begin{tabular}{|c|c|c|c|}
\hline Subsidiária como... & $\begin{array}{c}\text { Tipo 1 } \\
\text { Braço operacional }\end{array}$ & Unidade relativamente autônoma & $\begin{array}{c}\text { Tipo II } \\
\text { Centro de competências }\end{array}$ \\
\hline $\begin{array}{c}\text { Decisões de configuração } \\
\text { (papel da subsidiária } \\
\text { na estratégia global da } \\
\text { corporação) }\end{array}$ & $\begin{array}{c}\text { Processo de decisão } \\
\text { centralizado na matriz; } \\
\text { busca a racionalização global } \\
\text { das atividades }\end{array}$ & $\begin{array}{c}\text { Subsidiária local tem voz e um certo grau de } \\
\text { autonomia para gerenciar excedentes gerados } \\
\text { localmente }\end{array}$ & $\begin{array}{c}\text { Subsidiária local tem } \\
\text { poder para tomar } \\
\text { decisões }\end{array}$ \\
\hline Projeto de produto & $\begin{array}{c}\text { Padronização em } \\
\text { termos globais; pequena } \\
\text { customização local }\end{array}$ & $\begin{array}{c}\text { Se a TNC é líder, desenvolve inovações radicais e } \\
\text { projetos plataforma na matriz; projetos derivativos } \\
\text { são desenvolvidos localmente }\end{array}$ & $\begin{array}{c}\text { Liderança global } \\
\text { da subsidiária no } \\
\text { desenvolvimento de } \\
\text { Sertos produtos }\end{array}$ \\
\hline
\end{tabular}

Fonte: Adaptado de Fleury e Fleury (2000). 
Outro ponto relevante para completar esse referencial teórico trata das diferentes estruturas de DGP. Duas categorias principais de estruturas de trabalho foram identificadas na teoria em DGP (CHIESA, 2000). A primeira, denominada "estrutura baseada na especialização", uma determinada unidade de DP ou laboratório estrangeiro é designada como totalmente responsável para o desenvolvimento de um novo produto, processo ou tecnologia, baseado em um mandante global. 0 segundo tipo, "estrutura baseada na integração", defende que diferentes unidades contribuam com o programa de desenvolvimento de tecnologia e projetos e, como resultado desse trabalho conjunto, têm-se as inovações globais.

\subsection{Síntese do quadro teórico}

Cabe primeiramente destacar que existem outros fatores relacionados à estratégia competitiva e à economia (e.g. relevância e o porte dos mercados, aspectos associados à política e economia internacionais da produção, dentre outros) que também podem influenciar nas decisões quanto à descentralização do desenvolvimento de novos produtos e que não foram discutidos no referencial teórico anterior. Em função de que não seria possível estender o escopo do trabalho de modo a considerar a totalidade desses fatores e também pelo fato de existirem diferenças em termos de abrangência e hierarquia dos conceitos (por exemplo aqueles associados à política internacional e/ ou macroeconomia, que são mais genéricos ou amplos), alguns fatores foram selecionados para a presente investigação, caracterizando a delimitação do presente trabalho. Desse modo, a partir do referencial teórico anterior, decidiu-se investigar os fatores sintetizados no Quadro 3, que são então empregados como base para o trabalho de campo.
Como pode ser visto no Quadro 3, a literatura estudada (descrita anteriormente) pode ser basicamente reorganizada e dividida em três clusters distintos: processo de desenvolvimento de produto, o produto em si (com relação ao seu grau de inovação e de modularidade) e, por fim, a organização do desenvolvimento global de novos produtos. A lógica do quadro é a seguinte: a primeira coluna do quadro mostra um nível mais geral do cluster, seguido de um detalhamento de tópicos relativo a cada cluster na segunda coluna. Para cada tópico, os fatores nominados na terceira coluna são associados às principais referências que os apoiam. A análise empírica no objeto de estudo seguiu então essa estrutura como base do trabalho de pesquisa, cujos métodos empregados são discutidos na seção seguinte.

\section{Métodos de pesquisa}

0 presente trabalho pode ser classificado como de natureza exploratória, tendo em vista a premissa de que a literatura nesse tema não se encontra plenamente consolidada, principalmente no que refere às montadoras instaladas no país. Assim, busca atender o objetivo geral de analisar a descentralização de desenvolvimento de novos produtos, por meio de uma investigação das relações entre uma matriz alemã e sua subsidiária brasileira, atuante no setor de veículos comerciais, por meio da abordagem de estudo de caso, seguindo as orientações da literatura (YIN, 2001; VOSS et al., 2002).

Partindo-se da premissa que as subsidiárias brasileiras na indústria automobilística nacional estão envolvidas no DGP, já destacado em outras publicações (e.g. DIAS, 2003; CONSONI, 2004; WALLACE, 2004; DIAS; SALERNO, 2009) e a partir do objetivo geral anterior, este trabalho considera os seguintes objetivos específicos:

Quadro 3. Síntese do referencial teórico.

\begin{tabular}{|c|c|c|c|}
\hline Cluster & & Fatores identificados & Referências \\
\hline \multirow{2}{*}{$\begin{array}{l}\text { Processo de } \\
\text { desenvolvimento de } \\
\text { produto (PDP) }\end{array}$} & PDP & $\begin{array}{l}\text { Processo de desenvolvimento de produtos. } \\
\text { Revisão de fases, gates }\end{array}$ & $\begin{array}{l}\text { Cooper (1993) e Rozenfeld (1997) e } \\
\text { Rozenfeld et al. 2006) }\end{array}$ \\
\hline & $\begin{array}{l}\text { Gestão do portfólio de } \\
\text { projetos de produto }\end{array}$ & Processo de funil de desenvolvimento & $\begin{array}{l}\text { Clark e Wheelwright (1993) e } \\
\text { Rozenfeld et al. (2006) }\end{array}$ \\
\hline \multirow{2}{*}{$\begin{array}{l}\text { Produto - inovação e } \\
\text { modularidade }\end{array}$} & $\begin{array}{l}\text { Grau de inovação do } \\
\text { produto }\end{array}$ & $\begin{array}{l}\text { Projetos derivativos até projetos de } \\
\text { desenvolvimento avançado }\end{array}$ & $\begin{array}{l}\text { Clark e Wheelwright (1993), Consoni } \\
\text { (2004) e Dias (2003) }\end{array}$ \\
\hline & $\begin{array}{l}\text { Modularidade do } \\
\text { produto }\end{array}$ & $\begin{array}{l}\text { Produto modular; influência do produto } \\
\text { modular na descentralização ou não do DP }\end{array}$ & $\begin{array}{l}\text { Mikkola (2001), Dias (2003), } \\
\text { Pandremenos et al. (2009) e Cauchick } \\
\text { Miguel e Hsuan (2010) }\end{array}$ \\
\hline \multirow{3}{*}{$\begin{array}{l}\text { Organização do } \\
\text { desenvolvimento } \\
\text { global de produtos }\end{array}$} & $\begin{array}{l}\text { Autonomia em DP das } \\
\text { subsidiárias }\end{array}$ & $\begin{array}{l}\text { Tipos de estruturas de DGP e seus respectivos } \\
\text { graus de autonomia de decisão }\end{array}$ & $\begin{array}{l}\text { Behrman e Fischer (1980), Brockhoff } \\
\text { e Schmaul (1996), Chiesa (1999), } \\
\text { Ronstadt (1977), Reddy (1997) e Fleury } \\
\text { e Fleury (2000) }\end{array}$ \\
\hline & $\begin{array}{l}\text { Participação das } \\
\text { subsidiárias no DGP }\end{array}$ & $\begin{array}{l}\text { Modelo de "causas } \times \text { efeitos" e sua aplicação em } \\
\text { diferentes setores }\end{array}$ & $\begin{array}{l}\text { Galina e Plonski (2000), Dias e Galina } \\
\text { (2000) e Galina (2003) }\end{array}$ \\
\hline & Relações de poder & Relações de poder matriz e subsidiária & Dias (2003) \\
\hline
\end{tabular}


(i) identificar os principais fatores internos e externos que levam à descentralização do DGP (no contexto do setor de veículos comerciais) - já destacados no referencial teórico, considerando ainda como base um modelo conceitual proposto por Galina (2003) - e seus respectivos efeitos sobre a descentralização do DGP;

(ii) identificar e analisar como ocorre a relação entre a subsidiária e a matriz, como esta influencia a descentralização do DGP e contribui para a discussão da lacuna teórica apresentada por um estudo anterior (DIAS, 2003). Esse estudo anterior identifica a relação de poder entre a matriz e subsidiárias como uma lacuna na teoria sobre a organização de DGP, sobretudo com relação à decisão de descentralização do DGP. Como mostrado no Quadro 3, são considerados fatores que vão além da literatura básica - como no caso de fatores como a competência tecnológica e o baixo custo da mão de obra - mas também fatores como a relação de poder e história da subsidiária.

Por fim, busca ainda:

(iii) verificar se o projeto modular atua como agente facilitador da descentralização do DGP e, caso afirmativo, como este influencia na tomada de decisão de descentralização de projetos de novos produtos e, com isso, contribuir para a discussão da lacuna teórica apresentada por Dias (2003).

0 trabalho iniciou-se pelo levantamento bibliográfico sobre os temas relacionados ao desenvolvimento de produto e sua descentralização, anteriormente destacados. Por meio desta revisão, conseguiu-se identificar algumas lacunas na teoria sobre a descentralização de DGP no contexto da indústria automobilística e, assim, delimitar o problema de pesquisa. A revisão bibliográfica foi seguida de um trabalho de campo em uma organização do setor de veículos comerciais, sendo analisadas uma unidade de DP no Brasil e uma unidade de DP na matriz, localizada na Alemanha.

Os principais critérios para a escolha do setor industrial e da empresa objeto de estudo foram os seguintes: (i) a importância do objeto de análise no segmento de veículos comerciais no país e no mundo, (ii) a estrutura existente para o desenvolvimento de veículos comerciais da empresa, e (iii) a facilidade de acesso aos dados por um dos autores. Esses fatores, juntamente com um maior detalhamento do setor e das unidades de análise, são descritos na próxima seção.

\subsection{Setor de veículos comerciais e as unidades de análise}

No âmbito nacional, a indústria automotiva brasileira representa um dos mais proeminentes setores econômicos no país. 0 setor automotivo instalado é constituído por mais de vinte diferentes montadoras abastecidas por mais de quinhentas empresas de autopeças. Trata-se de um complexo industrial com capacidade instalada para produzir cerca de 4 milhões de veículos por ano e com um faturamento da ordem de mais de US\$ 100 bilhões, equivalente a 19,5\% do PIB industrial nacional (ASSOCIAÇÃO..., 2011). 0 volume anual de produção no setor automotivo vem crescendo de forma acentuada nos últimos anos. Em 2007, o volume produzido no Brasil alcançou a posição de sétimo maior produtor de veículos automotores do mundo (INTERNATIONAL..., 2008), estando mais recentemente na sexta posição segundo dados da Associação de Fabricantes de Veículos Automotores (ASSOCIAÇÃO..., 2011).

Em termos de investimentos em pesquisa e desenvolvimento (P\&D), estudos de Fransman (2001) mostram que a indústria automotiva mundial é um dos setores industriais que mais investem em P\&D (em média, cerca de 4,2\% do faturamento), depois da farmacêutica e de telecomunicações. No Brasil, estudos apoiados pelo Banco Nacional do Desenvolvimento - BNDES mostraram um investimento médio em atividades de P\&D pela indústria automobilística de cerca de 5\% sobre a sua receita. Similarmente, estudos de Oppat (2008) sobre a indústria automobilística alemã revelaram um investimento médio em P\&D por volta de 5\% sobre a receita bruta anual.

Dentro da indústria automobilística, um dos seus subsetores que se destacam pelo expressivo volume de produção em âmbito mundial é o segmento de veículos comerciais, responsável por cerca de 27\% do total de veículos produzidos no mundo em 2007 (INTERNATIONAL..., 2008, 2008). De acordo com a International Organization of Motor Vehicle Manufactures (INTERNATIONAL..., 2008), o Brasil atingiu em 2007 a nona posição no ranking mundial de produção de veículos comerciais (com pouco mais de 582 mil unidades produzidas), ultrapassando inclusive países como Alemanha (na décima posição, com uma produção pouco mais de 500 mil unidades), França, Itália e Turquia.

A empresa objeto de estudo encontra-se atualmente entre os cinco maiores produtores de veículos no mundo (INTERNATIONAL..., 2008). No segmento de veículos comerciais, a empresa encontra-se entre os sete maiores produtores mundiais com mais de $730 \mathrm{mil}$ unidades produzidas em 2010 (ASSOCIAÇÃO..., 2011). Em termos de DP de veículos comerciais, a empresa atua em três continentes desenvolvendo caminhões, ônibus e comerciais leves, com mais de cinco marcas nesses três segmentos. Possui ao todo um grupo de profissionais de DP com mais de 5.000 colaboradores, entre técnicos, engenheiros, físicos e gestores atuando em desenvolvimento de veículos comerciais e seus motores e câmbios. Na ocasião do presente estudo, 
a empresa possuía mais de 25 projetos de novos produtos em andamento (considerando-se todas as unidades de veículos comerciais e continentes) com um investimento anual em DP maior que 250 milhões de euros.

A subsidiária brasileira possui atividades de DP de caminhões, ônibus e power train (conjunto motor-câmbio-transmissão). Essas atividades iniciaram em meados da década de 1970, sendo intensificadas, sobretudo, a partir do início da década de 1980. Atualmente, possui cerca de 500 profissionais atuando em DP, dos quais cerca de 80 atuando em projetos conjuntos com a unidade matriz. Uma classificação dos projetos realizados pela subsidiária brasileira nos últimos dez anos é ilustrada no Quadro 4.

Os projetos classificados como "grande" constituem-se, sobretudo, de introduções de uma nova geração de produtos ou plataforma para o mercado nacional ou da América Latina. No entanto, a análise documental mostrou que essa "nova geração" para o mercado local brasileiro é, na verdade, oriunda de derivativos ou adaptações dos produtos europeus para as necessidades locais. Em geral, o nível de adaptação varia entre a nacionalização de peças até a tropicalização de componentes para as condições locais, como por exemplo partes da suspensão, reforço de chassis e simplificação do acabamento interno.

\subsection{Coleta e análise de dados}

Para a coleta dos dados, foram estruturados instrumentos (roteiros semiestruturados e questionários), bem como foram utilizadas outras fontes de evidência, tais como a análise documental e a observação direta. A coleta de dados estendeu-se por volta de 6 meses, considerando a necessidade de obtenção de dados in loco em ambos os países. Um questionário foi aplicado na unidade de DP brasileira e na matriz para executivos em DP, sendo este baseado em diversas fontes da literatura sobre a caracterização e descentralização do DGP. 0 questionário foi, ainda, resultado também de um processo de iteração, sendo aplicado primeiramente a uma amostra controlada de seis executivos na forma de "piloto". No total, foram distribuídos 30 questionários por meio eletrônico para executivos em DP da matriz e na subsidiária brasileira (gerência, gerência sênior e diretoria), obtendo-se uma taxa de retorno de 63\%. Além do questionário, utilizou-se de entrevistas semiestruturadas com gerentes seniores de DP em ambas as unidades. Foram realizadas ao todo quatro entrevistas, sendo duas em cada unidade.

A análise documental levantou registros relevantes relacionados ao PDP de ambas as unidades de DP, como por exemplo: (i) portfólio de projetos de novos produtos (sobretudo caminhões) da matriz e subsidiária brasileira, atual e dos últimos cinco anos; (ii) planejamento estratégico de lançamento de novos produtos dos últimos cinco anos (por uma questão de confidencialidade, detalhes necessitam ser omitidos); (iii) definição do escopo dos últimos três projetos realizados em conjunto entre matriz e subsidiária brasileira (verba de engenharia, organograma, número de pessoal técnico envolvido, estrutura e tipo de produto, grau de inovação do produto ou família de produtos); (iv) atas de reuniões de revisão de projetos de dois projetos de produtos realizados nos últimos cinco anos; (v) documentos organizacionais de ambas as unidades de DP, como organogramas, e métodos de trabalho (PDP).

Quadro 4. Classificação dos projetos realizados pela subsidiária brasileira nos últimos dez anos.

\begin{tabular}{|c|c|c|c|c|c|}
\hline $\begin{array}{l}\text { Tipo de } \\
\text { projeto }\end{array}$ & Projeto típico encontrado & $\begin{array}{l}\text { Verba de engenharia } \\
\text { (média, em milhões } \\
\text { de euros) }\end{array}$ & $\begin{array}{l}\text { Duração média } \\
\text { (em anos) }\end{array}$ & $\begin{array}{l}\text { Quantidade de projetos } \\
\text { em andamento em } \\
\text { paralelo por segmento }\end{array}$ & $\begin{array}{l}\text { Número de reuniões de } \\
\text { revisões de fases (QGs) }\end{array}$ \\
\hline Grande & $\begin{array}{l}\text { Plataforma ou próxima } \\
\text { geração para o Brasil, ou } \\
\text { participação em projeto } \\
\text { da matriz }\end{array}$ & Entre 20 e 100 & $\begin{array}{l}3 \text { a } 4 \text { anos. QG } \\
\text { de fechamento } 1 \\
\text { ano após início } \\
\text { da produção }\end{array}$ & Máximo 2 & $\begin{array}{c}11 \text {, sendo as revisões de } \\
\text { fases com a participação } \\
\text { da matriz }\end{array}$ \\
\hline Médio & $\begin{array}{c}\text { Derivativos ou } \\
\text { incrementais: alterações de } \\
\text { estilo, redução de custo, } \\
\text { introdução de um ou } \\
\text { dois elementos de nova } \\
\text { tecnologia em relação ao } \\
\text { produto atual }\end{array}$ & Entre cerca de 11 e 20 & $\begin{array}{l}1 \text { a } 2,5 \text { anos. QG } \\
\text { de fechamento } 6 \\
\text { meses após início } \\
\text { da produção }\end{array}$ & Máximo 3 & $\begin{array}{l}5 \text { a } 7, \text { podendo variar } \\
\text { conforme decisão do } \\
\text { gerenciamento do projeto } \\
\text { junto à presidência da } \\
\text { unidade Brasil }\end{array}$ \\
\hline Pequeno & $\begin{array}{c}\text { Derivativos ou } \\
\text { incrementais: redução } \\
\text { de custo, introdução de } \\
\text { uma variante nova ou } \\
\text { um elemento de nova } \\
\text { tecnologia }\end{array}$ & Até cerca de 10 & $\begin{array}{l}0,5 \text { a } 1 \text { ano. QG } \\
\text { de fechamento } \\
\text { junto ao início da } \\
\text { produção }\end{array}$ & Cerca de 5 & 4 \\
\hline
\end{tabular}


A observação direta ocorreu da interação direta entre o pesquisador e os envolvidos no PDP, por meio de visitas às unidades e áreas envolvidas na condução do PDP. 0 acompanhamento da condução de projetos de novos produtos ocorreu por meio de participação em reuniões e fóruns de desenvolvimento e gerenciamento de portfólio de projetos, ao qual um dos autores teve pleno acesso. No entanto, foram tomados cuidados para atenuar a influência daquele autor que faz parte do objeto de estudo, mas também pelo caráter de sigilo de parte dos dados acessados. Para minimizar a influência do pesquisador, recomendações da literatura (e.g. YIN, 2001; VOSS et al., 2002) foram levadas em consideração, além de análise crítica feita pelo coautor do presente trabalho, principalmente no que se refere à análise dos dados. Para a condução dessa análise, os dados foram compilados e transcritos em busca de uma análise de conteúdo. As diferentes fontes de evidências foram então confrontadas, buscando identificar convergência e/ou divergência, usando técnicas de triangulação.

\section{Resultados: análise do DGP da empresa investigada}

Primeiramente, realizou-se uma sintese comparativa das unidades de DP investigadas e seu posicionamento perante a teoria vigente, sobretudo quanto ao PDP, a gestão do portfólio de projetos, o grau de inovação associado aos projetos de produto e, por fim, o grau de autonomia em DP da subsidiária. 0 Quadro 5 sintetiza tais resultados.

A autonomia em desenvolvimento de novos produtos da subsidiária brasileira está diretamente ligada ao seu papel no contexto global de DP da corporação. A subsidiária de caminhões pode ser classificada como uma unidade de "DP de adaptação", onde seu papel principal é gerar projetos para atender o mercado latino-americano e, na maioria das vezes, na atualidade, esses projetos são derivativos dos produtos europeus. Essa unidade pode ser enquadrada no tipo 11 (unidade relativamente autônoma) proposto por Fleury e Fleury (2000), no que tange ao projeto de produto.

A unidade de DP de ônibus possui, no entanto, um papel claro de ser a responsável por desenvolvimento de chassis de ônibus mundial na organização de DGP. Isso significa que ela atende mercados como Ásia, África e Europa oriental, além do latino-americano, possuindo um papel especializado num processo de DGP coordenado centralmente. Assim, essa unidade pode ser caracterizada como uma unidade de "DP originalmente global" e tende para o tipo 111 (centro de competência) na estrutura proposta por Fleury e Fleury (2000). Tais diferentes papéis na estrutura de DGP levam a diferentes graus de autonomia das diretorias de DP brasileiras: a de ônibus, com maior autonomia, pode ser classificada no modelo de Chiesa (1999) como uma estrutura "controle baseado em recursos" e a de caminhões, com menor autonomia, podendo ser enquadrada na categoria "centralização com decisão participativa”.

Os tópicos identificados na literatura e sintetizados no Quadro 3 ("participação das subsidiárias no DGP”, "relações de poder" e "modularidade do produto") são discutidos em mais detalhes nas seções seguintes.

Quadro 5. Síntese comparativa da caracterização das unidades de DP estudadas.

\begin{tabular}{|c|c|c|c|c|}
\hline & Unidade matriz & Subsidiária brasileira & $\begin{array}{l}\text { Literatura base ou lacuna } \\
\text { encontrada }\end{array}$ & Considerações \\
\hline PDP & $\begin{array}{c}\text { Revisão de fases, } \\
\text { Quality Gates }\end{array}$ & $\begin{array}{l}\text { ldem à matriz, sem } \\
\text { adaptação local }\end{array}$ & $\begin{array}{c}\text { Cooper (1993) e Rozenfeld } \\
(1997,2006)\end{array}$ & \multirow{2}{*}{$\begin{array}{c}\text { Nenhuma lacuna significante } \\
\text { encontrada com base na literatura. } \\
0 \text { modelo adotado pela empresa se } \\
\text { adere à literatura vigente }\end{array}$} \\
\hline $\begin{array}{l}\text { Gestão do } \\
\text { portfólio de } \\
\text { projetos }\end{array}$ & $\begin{array}{c}\text { Funil de } \\
\text { desenvolvimento }\end{array}$ & $\begin{array}{l}\text { ldem à matriz, com controle } \\
\text { da matriz para projetos de } \\
\text { "médio e grande porte" }\end{array}$ & $\begin{array}{l}\text { Clark e Wheelwright (1993) } \\
\text { e Rozenfeld et al. (2006) }\end{array}$ & \\
\hline $\begin{array}{l}\text { Grau de inovação } \\
\text { do produto }\end{array}$ & $\begin{array}{l}\text { Próxima geração } \\
\text { ou plataforma; } \\
\text { incrementais ou } \\
\text { derivativos }\end{array}$ & $\begin{array}{c}\text { Normalmente incrementais } \\
\text { ou derivativos do produto } \\
\text { europeu }\end{array}$ & $\begin{array}{c}\text { Geralmente próxima } \\
\text { geração ou plataforma; } \\
\text { incrementais ou derivativos } \\
\text { (CONSON1, 2004; DIAS, } \\
\text { 2003) }\end{array}$ & $\begin{array}{l}\text { Subsidiária brasileira pode ser } \\
\text { enquadrada no tipo } 11 \text { “Unidade } \\
\text { relativamente autônoma” quanto } \\
\text { ao projeto de produto (FLEURY; } \\
\text { FLEURY, 2000) }\end{array}$ \\
\hline $\begin{array}{l}\text { Autonomia em } \\
\text { DP da subsidiária }\end{array}$ & & $\begin{array}{l}\text { Variável ao longo dos últimos } \\
20 \text { anos. Hoje: caminhões } \\
\text { com autonomia somente } \\
\text { para projetos pequenos; } \\
\text { ônibus com maior autonomia } \\
\text { por ser centro de excelência } \\
\text { mundial em chassis }\end{array}$ & $\begin{array}{c}\text { Behrman e Fischer (1980), } \\
\text { Brockhoff e Schmaul } \\
\text { (1996), Chiesa (1999), } \\
\text { Ronstadt (1977), Reddy } \\
\text { (1997) e Fleury e Fleury } \\
\text { (2000) }\end{array}$ & $\begin{array}{l}\text { Possível relação entre o papel da } \\
\text { unidade subsidiária (CHIESA, 1995, } \\
\text { 1999: PeD de adaptação versus PeD } \\
\text { originalmente global) e o grau de } \\
\text { autonomia delegado pela matriz }\end{array}$ \\
\hline
\end{tabular}




\subsection{Participação da subsidiária brasileira no DGP - modelo de "causa x efeito"}

Quanto à análise da aderência ao modelo de "causa x efeito" sobre a descentralização do DGP, utilizou-se das respostas advindas dos questionários, considerando-se a frequência das mesmas por tipo de efeito. A descrição dos efeitos é uma tentativa de mensurar a presença de um efeito/decorrência ("existe" ou "não existe"), intensidade ("alta" ou "baixa"), e nível de exigência ou estratégico. Parte dos resultados do quadro, no que tange à escala Lickert de 1 a 5 utilizada, é proveniente dos resultados dos questionários, sendo que a análise destes foi complementada pelos resultados das entrevistas. Os resultados, juntamente com uma análise crítica dos autores (na coluna dos "comentários"), podem ser vistos no Quadro 6.

Em suma, pode-se constatar que causas de alocação de atividades de DP mais relevantes no caso da empresa objeto de estudo foram o "interesse no mercado brasileiro e latino americano" e os "aspectos históricos” deste e da evolução da subsidiária brasileira. Diferentemente do esperado, esses fatores parecem estar à frente de questões como o custo de DP local e da competência da subsidiária, pois tal resultado diverge da literatura já relativamente estabelecida sobre descentralização de DP (CHIESA, 1995; REDDY, 1997). Além disso, esses dados reforçam o estudo de Dias
(2003) e Dias e Salerno (2009), os quais demonstram que as subsidiárias brasileiras de montadoras de veículos (e.g. Fiat, GM e VW) levam em conta de maneira relevante os fatores das relações de poder entre matriz e subsidiária e a evolução histórica desta última. Fleury e Fleury (2000) destacam também a importância do interesse sobre o mercado local quanto às relações da matriz e subsidiárias.

Diferentemente do setor de telecomunicações analisado da literatura (GALINA; PLONSKI, 2000; GALINA, 2003), fatores como incentivos fiscais e atendimento às exigências governamentais locais para regulamentação apresentam um papel bem menos importante para o setor de veículos comerciais. Incentivos fiscais específicos para o DP de veículos comerciais não foram identificados, fato que vem ao encontro das conclusões de Dias (2003) quanto ao setor automotivo nacional de que agentes externos à empresa, em geral, não influenciaram a estrutura de DGP. Algumas iniciativas de agentes externos, tais como o incentivo a veículos propulsionados por motores de 1.000 cilindradas ou o incentivo ao uso de combustível alternativo, foram identificadas. Porém, a existência destas não garante que o desenvolvimento ou adaptação dos produtos serão gerados localmente. Além destas, identificaram-se apenas o "Índice de Conteúdo Regional" (ICR) e a "Lei do Bem" (BRASIL, 2005) como incentivos a um desenvolvimento local. 0 ICR leva em conta que um

Quadro 6. Aplicação do modelo de "causa x efeito" na empresa objeto de estudo.

\begin{tabular}{|c|c|c|}
\hline Causas & Descrição dos efeitos & Comentários \\
\hline $\begin{array}{l}\text { Interesse no mercado } \\
\text { brasileiro e latino }\end{array}$ & $\begin{array}{c}\text { Alto }-\mathrm{P} \\
\text { Baixo }-\mathrm{NP}\end{array}$ & $\begin{array}{l}\text { A importância do mercado e respectiva unidade de negócios influenciam a alocação } \\
\text { de DP no Brasil }\end{array}$ \\
\hline Competências locais & $\begin{array}{c}\text { Existe }-\mathrm{P} \\
\text { Não existe }-\mathrm{NP}\end{array}$ & $\begin{array}{l}\text { Visão da subsidiária difere da matriz. Subsidiária supervaloriza suas competências, } \\
\text { enquanto matriz valoriza apenas competências de DP para mercados de países em } \\
\text { desenvolvimento }\end{array}$ \\
\hline $\begin{array}{l}\text { Custo de desenvolvi-mento } \\
\text { local }\end{array}$ & $\begin{array}{l}\text { Baixo - P } \\
\text { Alto - NP }\end{array}$ & $\begin{array}{l}\text { Baixo custo de DP em relação à matriz influencia a decisão de alocação de DP no Brasil, } \\
\text { sobretudo para caminhões. A unidade de ônibus, na forma de centro de excelência, } \\
\text { atribui menor peso para a questão de custos de DP }\end{array}$ \\
\hline Autonomia da subsidiária & $\begin{array}{c}\text { Existe }-\mathrm{P} \\
\text { Não existe }-\mathrm{NP}\end{array}$ & $\begin{array}{l}\text { Especialmente relevante para o DP ônibus, o qual atua como estrutura especializada, } \\
\text { tipo } 111 \text { (FLEURY; FLEURY, 2000). Para caminhões, que atuam em estrutura do tipo de } \\
\text { integração, a relevância é bem menor: estrutura do tipo } 11 \text { (FLEURY; FLEURY, 2000) }\end{array}$ \\
\hline Incentivos fiscais & $\begin{array}{c}\text { Existe }-\mathrm{P} \\
\text { Não existe }-\mathrm{NP}\end{array}$ & $\begin{array}{l}\text { Incentivos fiscais para o DP automotivo no Brasil não constitui fator decisivo na } \\
\text { alocação de atividades de DP no país pela empresa estudada }\end{array}$ \\
\hline Tipo de produto & $\begin{array}{l}\text { Estratégico } \\
\text { localmente - P } \\
\text { Não estratégico } \\
\text { localmente - NP }\end{array}$ & $\begin{array}{l}0 \text { fato da necessidade de se adaptar os produtos europeus ao mercado local, para } \\
\text { caminhões, mostra a importância de tal fator. Para ônibus, a importância é ainda maior, } \\
\text { pois gerar seus próprios produtos que são estratégicos não somente localmente, mas } \\
\text { para outros mercados }\end{array}$ \\
\hline Aspectos históricos & $\begin{array}{l}\text { Favorável ao DP - P } \\
\text { Não favorável ao } \\
\text { DP - NP }\end{array}$ & $\begin{array}{l}0 \text { desenvolvimento histórico do mercado automotivo local e do papel da subsidiária } \\
\text { neste, bem como nas relações de DP com a matriz, mostra a alta importância dessa } \\
\text { causa de alocação de atividades de DP no Brasil }\end{array}$ \\
\hline Exigências governamentais & $\begin{array}{l}\text { Exigências para } \\
\text { adaptações - P } \\
\text { Não exigência - NP }\end{array}$ & $\begin{array}{l}\text { A subsidiária vê a questão do atendimento das normas locais como primordial para as } \\
\text { atividades de DP. Na visão da matriz, a qual possui produtos tecnologicamente mais } \\
\text { avançados, esta poderia suprir tais exigências legais sem a necessidade de adaptações } \\
\text { locais na subsidiária }\end{array}$ \\
\hline
\end{tabular}

Efeitos para a subsidiária: ${ }^{*} \mathrm{P}=$ alta probabilidade de participar do DGP; ${ }^{* *} \mathrm{NP}$ baixa probabilidade de participar no DGP**** $=$ classificação obtida mediante entrevistas, para causas e respectivos efeitos (média). Notas atribuídas em uma escala de 1 a 5 , onde: $5=$ muito relevante; 1 = irrelevante. 
produto automotivo é considerado como local se tal índice de nacionalização for maior ou igual a 60\% (BRASIL, 2006), recentemente atualizado para 65\% (BRASIL, 2011). Se isso ocorrer, incentivos na forma de financiamento para as facilidades de produção de tais produtos são concedidos pelo governo ou entidades financeiras regulamentadas. No entanto, tal incentivo não implica em DP necessariamente, podendo se limitar a um processo de nacionalização do original estrangeiro. A "Lei do Bem" consolidou os incentivos fiscais que as pessoas jurídicas podem usufruir de forma automática desde que realizem pesquisa tecnológica e desenvolvimento de inovação tecnológica. Os principais incentivos oferecidos pela "Lei do Bem" constituem, sobretudo, deduções de imposto de renda e da contribuição sobre o lucro líquido de dispêndios efetuados em atividades de pesquisa e desenvolvimento; redução do imposto de renda retido na fonte incidente sobre remessa ao exterior, resultantes de contratos de transferência de tecnologia.

No entanto, nas entrevistas realizadas, a "Lei do Bem" não foi citada como fator relevante para a participação da filial brasileira no DGP, sendo a causa "incentivos fiscais" classificada como irrelevante na visão dos entrevistados. A análise documental de engenharia dos projetos realizada em conjunto com a matriz nos últimos três anos também não identificou tal fator como relevante para a distribuição de atividades de DP.

Quanto ao atendimento das exigências governamentais para a regulamentação, estas são, sobretudo, relacionadas aos níveis de emissões $\left(\mathrm{CO}_{2}\right.$ e particulado) e ruído, ambas regulamentadas pelo Conama (Conselho Nacional de Meio Ambiente). No entanto, os produtos advindos da Europa já atendem essas normas, pois, em geral, encontram-se uma geração à frente em termos de exigências ambientais.

Assim, pode-se constatar que, com exceção dos fatores de regulamentação governamental e incentivos fiscais, o modelo de "causa $\times$ efeito" proposto por Galina (2003) para o setor de telecomunicações aparentemente pode retratar também o setor de veículos comerciais quanto aos fatores de atração de atividades de DGP para o Brasil e seus efeitos. No entanto, é necessária certa cautela, pois os setores industriais são diferentes, restringindo a explicação do fenômeno com o referido arcabouço teórico. Além disso, o modelo não atribui diferentes pesos às causas da participação do DGP, focando Galina (2003), em suas conclusões, sobretudo nos incentivos fiscais oferecidos para o setor de telecomunicações como atrativo de atividades de DP para o Brasil. Tal fato constitui uma limitação do modelo que fica evidente no presente trabalho e pode ser base para trabalhos futuros.

\subsection{Decisão de descentralização do DGP - Influências das relações entre matriz e subsidiária brasileira}

Um elemento importante para se definir se o DGP será mais ou menos descentralizado e quais as subsidiárias serão envolvidas nessas atividades é a relação de poder existente entre a matriz, o centro de projeto e as subsidiárias (DIAS, 2003). As filiais não são sujeitos passivos no processo de definição da divisão internacional do trabalho de DP, atuando de forma ativa a fim de atrair para si atividades de DP, por meio do aumento da capacitação de seus funcionários, realização de parcerias com institutos de pesquisa locais e buscando financiamentos (DIAS; SALERNO, 2009).

Como resultados principais, observou-se que fatores tais como o "papel ativo da subsidiária", a "evolução histórica desta em termos de DP" e a "relação de confiança estabelecida com a matriz" apresentam um forte papel na decisão de descentralização do DGP. A análise das diferentes fontes de evidência na subsidiária brasileira mostrou que a mesma apresentou um papel ativo no início dos anos 2000, a fim de atrair atividades de DGP para si, buscando atividades de desenvolvimento de produto em conjunto com a matriz (por exemplo, desenvolvimento de partes ou componentes específicos de um projeto da matriz, ou, ainda, testes de validação e geração e implementação de melhorias de tais componentes). Convergentemente, tal fato ficou evidente não apenas de acordo com os entrevistados da unidade subsidiária brasileira, mas também com a análise documental dos últimos três projetos de novos produtos realizados pela unidade brasileira em conjunto com a matriz.

Foi possível levantar que, para a diretoria de caminhões da unidade brasileira, tal iniciativa resultou em participação em dois projetos de médio porte e um projeto de grande porte da matriz nos últimos cinco anos, sobretudo desenvolvendo módulos de cabinas e chassis. Mais de 80 profissionais brasileiros de DP foram envolvidos nesse processo, gerando uma receita para a unidade brasileira de cerca de 50 milhões de euros nos últimos cinco anos. Para a diretoria de ônibus, a consolidação do centro de excelência é considerada como resultado de tal processo. Tais constatações induzem a afirmar que o segmento de veículos comerciais reage similarmente ao de veículos de passeio, demonstrado em trabalhos anteriores (DIAS; GALINA, 2000; DIAS, 2003; DIAS; SALERNO, 2009). No entanto, deve-se considerar que esse fato ocorre em alguns (poucos) pontos específicos, pois, embora pertencentes ao mesmo setor econômico, os segmentos são relativamente diferentes em termos de mercado, forma de desenvolver produtos, realização de negócios, aspectos legais etc. 
Quadro 7. Proposta de relação entre tipo de estrutura de DGP e projeto modular.

\begin{tabular}{|c|c|c|c|}
\hline \multicolumn{2}{|c|}{$\begin{array}{l}\text { Estrutura de DGP } \\
\text { (CHIESA, 2000) }\end{array}$} & Empresa estudada & Projeto modular facilita a descentralização do DGP? \\
\hline \multirow{2}{*}{$\begin{array}{l}\text { Baseada na } \\
\text { especialização }\end{array}$} & $\begin{array}{l}\text { do tipo centro de } \\
\text { excelência }\end{array}$ & DP ônibus & $\begin{array}{l}\text { Não, a estrutura do produto não interfere no processo } \\
\text { de estruturação de DGP }\end{array}$ \\
\hline & $\begin{array}{l}\text { do tipo suporte } \\
\text { especializado }\end{array}$ & Não encontrada & $\begin{array}{l}\text { Não se aplica ao caso estudado, não sendo possível } \\
\text { concluir }\end{array}$ \\
\hline \multirow{2}{*}{$\begin{array}{l}\text { Baseada na } \\
\text { integração }\end{array}$} & do tipo rede & $\begin{array}{l}\text { DP caminhões para projetos } \\
\text { grandes da subsidiária }\end{array}$ & $\begin{array}{l}\text { Sim, principalmente quanto à transferência de } \\
\text { tecnologia da matriz para a subsidiária (derivativos } \\
\text { adaptados para o mercado local) }\end{array}$ \\
\hline & $\begin{array}{l}\text { do tipo contribuinte } \\
\text { especializado }\end{array}$ & $\begin{array}{l}\text { DP caminhões para projetos } \\
\text { grandes realizados em conjunto } \\
\text { entre matriz e uma ou mais } \\
\text { subsidiárias }\end{array}$ & $\begin{array}{l}\text { Sim, principalmente quanto a uma clara divisão de } \\
\text { responsabilidades em "pacotes de trabalho" a serem } \\
\text { desenvolvidos por cada unidade de DP envolvida no } \\
\text { projeto. }\end{array}$ \\
\hline
\end{tabular}

\subsection{Decisão de descentralização do DGP - Influências dos projetos em módulos}

Com relação à análise da influência do projeto em módulos no processo de descentralização do DGP, verificou-se uma forte diferença entre o posicionamento dos executivos das diretorias de desenvolvimento de ônibus e caminhões. Para a diretoria de DP de caminhões, mais de 70\% dos respondentes acreditam que projetos em módulos influenciam positivamente a decisão de descentralização de DGP. Para a diretoria de ônibus, no entanto, cerca de $87 \%$ dos respondentes dizem que o projeto em módulo não influencia a descentralização do DGP. Esse fato pode ser explicado quando se somam as entrevistas e análise documental realizadas em ambas as unidades, levando à conclusão que o projeto em módulos influencia na decisão de descentralização de DGP, dependendo do tipo de estrutura de DGP adotado. 0 Quadro 7 exibe uma proposta dos autores quanto à relação entre tipo de estrutura de DGP e projeto modular.

Pode-se observar que, para estruturas do tipo de especialização ou centro de excelência (CHIESA, 2000), onde a subsidiária é responsável pelo desenvolvimento de um determinado tipo de produto específico do portfólio da empresa, o projeto em módulos parece exercer influência nula nessa decisão. Este é o caso da diretoria de DP de ônibus da empresa estudada, visto que por esta foi adotado o conceito de "centro de excelência” em chassis de ônibus, não havendo influência da estrutura do produto na organização ou distribuição de atividades de DP entre matriz e subsidiária. Essa conclusão depende obviamente do nível de divisão de módulos adotado, ou seja, no presente caso considerando como módulo as subpartes de chassis, e não este como um módulo de um ônibus completo.

No entanto, para estruturas do tipo colaborativas ou de integração (CHIESA, 2000), onde as subsidiárias apresentam um papel de desenvolvimento em conjunto, gerando parte de um projeto ou pesquisa, o projeto em módulos parece ser um fator importante no processo de decisão de distribuição e coordenação do DGP. 0 uso de projetos modulares fica evidenciado na diretoria de DP caminhões, onde a possibilidade de a matriz delegar o desenvolvimento de módulos, sobretudo de cabinas e chassis de caminhões, ficou clara e colaborou com o processo de descentralização do DGP. Tais constatações indicam um passo além ao estudo de Dias (2003) realizado nas subsidiárias brasileiras da GM, Fiat e VW, a qual não identificou o projeto modular como um facilitador da descentralização do DGP.

\section{Conclusões}

Com relação à aplicação do modelo de "causa $\times$ efeito", pode-se concluir que, exceto pela questão da regulamentação governamental e incentivos fiscais, aquele modelo utilizado no setor de telecomunicações pode retratar também o setor de veículos comerciais quanto aos fatores de atração de atividades de DGP para o Brasil e seus efeitos. Dessa maneira, pode não haver necessidade para a proposição de um novo modelo para explicar as relações de descentralização do DGP para o setor de veículos comerciais da indústria automobilística. No entanto, necessita-se que estudos empíricos subsequentes com uma quantidade maior de unidades de análise sejam realizados para validar tal pressuposto apoiado, por enquanto, apenas no estudo de caso único da organização objeto de estudo.

Quanto ao fato de como a relação de poder entre a matriz e a subsidiária influencia a decisão de descentralização do DGP, foi identificado que os fatores com maior relevância para a decisão de descentralização do DGP são: o "papel ativo da subsidiária", a "evolução histórica desta em termos de DP" e a "relação de confiança estabelecida com a matriz". Isso leva à conclusão de que um papel ativo da subsidiária na busca por projetos junto à matriz e sua evolução em termos de relação de confiança com a matriz são, muitas vezes, mais importantes que 
fatores como capacitação em línguas ou entendimento da cultura da outra parte envolvida no processo de descentralização do DGP. Tal fato colabora com o aumento do corpo teórico dos estudos sobre as subsidiárias alocadas em países em desenvolvimento como agentes da descentralização do DGP, o qual, como previsto, pode ser considerado ainda como incipiente na literatura. Diferentemente do esperado, os interesses do mercado nacional não foram identificados como relevantes, na visão da organização investigada. Aparentemente, o ponto de vista mais importante é interno à organização, uma vez que os fatores com maior relevância são internos. No entanto, as razões para esse resultado não puderam ser devidamente explicadas, resultando em uma limitação do presente trabalho, que necessitaria de um aprofundamento investigativo.

No caso do terceiro objetivo específico do trabalho, que questiona se o projeto em módulos facilita a descentralização do desenvolvimento de produtos e como isso ocorre, pode-se concluir que o projeto em módulos influencia na decisão de descentralização de DGP dependendo do tipo de estrutura de DGP adotado. Esta relação, que não foi encontrada na literatura, pode ser considerada como a principal contribuição do presente trabalho. Pode-se concluir que para estruturas do tipo de "especialização" ou "centro de excelência” o projeto em módulos parece exercer influência praticamente nula nessa decisão, dependendo obviamente do nível de divisão de módulos adotado. No entanto, para estruturas do tipo "colaborativas" ou "de integração", onde as subsidiárias apresentam um papel de desenvolvimento em conjunto com a matriz, o projeto em módulos parece ser um fator importante no processo de decisão de distribuição e coordenação do DGP entre unidades de uma mesma empresa. Tal constatação vai um passo além dos estudos realizados nas subsidiárias brasileiras em outras montadoras, os quais não identificaram o projeto modular como um facilitador da descentralização do DGP numa mesma organização, apenas em projetos realizados em conjunto com fornecedores.

Como continuidade ao presente trabalho, sugere-se uma investigação mais ampla (estendendo a quantidade de objetos de análise) e aprofundada sobre a influência do produto modular no processo de descentralização do DGP. Além da análise de produtos modulares, trabalhos futuros com foco no nível operacional do processo do DPG podem ser realizados a fim de se aprofundar o tópico, como por exemplo: fatores relevantes para a manutenção do DGP e seus graus de importância para matriz e subsidiária ou, ainda, a influência da evolução das ferramentas computacionais de DP no processo de descentralização deste. Ademais, a investigação sobre o processo de descentralização do
DGP no setor de veículos comerciais pode ser ampliada em termos de amostra e, por fim, correlacionada com outros subsetores do segmento automotivo, como o de autopeças.

\section{Agradecimentos}

Os autores sinceramente agradecem aos avaliadores da Revista Produção pelas contribuições para a melhoria do presente trabalho. Um dos autores participa como colaborador no Programa de Pós-Graduação em Engenharia de Produção da Universidade de São Paulo, mas tem vínculo atual de trabalho com a Universidade Federal de Santa Catarina (UFSC), razão pela qual esta instituição também merece agradecimentos. Finalmente, os autores agradecem à empresa na qual o estudo foi realizado. Porém, cabe destacar que os resultados não representam a posição da organização investigada, mas sim a interpretação dada pelos autores.

\section{Referências}

ASSOCIAÇÃO NACIONAL DOS FABRICANTES DE VEÍCULOS AUTOMOTORES - ANFAVEA. Anuário da Indústria Automobilística Brasileira. São Paulo: ANFAVEA, 2011. Disponivel em: <http://www.anfavea.com.br>. Acesso em: 26 set. 2011.

BALDWIN, C. Y.; CLARK, K. Managing in the ages of modularity. Boston: Harvard Business Review, 1997.

BARTLETT, C. A.; GHOSHAL, S. Managing across borders. Boston: Harvard Business School Press, 1989.

BÉLIS-BERGOUIGNAN, M.; BORDENAVE, G.; LUNG, Y. Global strategies in the automobile industry. Actes Du Gerpisa, n. 18, p. 99-115, 1996.

BEHRMAN, J. N.; FISCHER, W. A. Overseas ReD activities of transnational companies. Cambridge: Ölgeschlager, Gunn e Hain, 1980.

BRASIL. Presidência da República Casa Civil. Subchefia para Assuntos Jurídicos. Lei 11.196, de 21 de novembro de 2005. Lei do Bem. Diário Oficial da República Federativa do Brasil, Brasília, DF, 22 nov. 2005. Seção 1, p. 1. Disponível em: <http://www.planalto.gov.br/ ccivil_03/_ato2004-2006/2005/lei/111196.htm>. Acesso em: 29 set. 2011.

BRASIL. Presidência da República Casa Civil. Subchefia para Assuntos Jurídicos. Decreto $n^{\circ} 6.006$, de 28 de dezembro de 2006. Aprova a Tabela de Incidência do Imposto sobre Produtos Industrializados - TIPI. Diário Oficial da República Federativa do Brasil, Brasília, DF, 29 dez. 2006. Disponível em: <http://www.planalto. gov.br/CCIVlL_03/_Ato2004-2006/2006/Decreto/ D6006.htm>. Acesso em: 29 set. 2011.

BRASIL. Presidência da República Casa Civil. Subchefia para Assuntos Jurídicos. Decreto $n^{\circ} 7.567$, de 15 de setembro de 2011. Regulamenta os arts. $5^{\circ}$ e $6^{\circ}$ da Medida Provisória $n^{\circ} 540$, de 2 de agosto de 2011 , os quais dispõem sobre a redução do Imposto sobre Produtos Industrializados - IPI em favor da indústria automotiva, e altera a Tabela de Incidência do IPI - TIPl, aprovada pelo Decreto $n^{\circ} 6.006$, de 28 de dezembro de 2006 . Diário Oficial da República Federativa do Brasil, Brasília, DF, 16 set. 2011. Disponível em: <http://www.planalto. 
gov.br/CCIVIL_03/_Ato2011-2014/2011/Decreto/D7567. htm>. Acesso em: 29 set. 2011.

BROCKHOFF, K. K. L.; SCHMAUL, B. Organization, autonomy, and success of internationally dispersed R\&D facilities. IEEE Transactions on Engineering Management, v. 43, n. 1, p. 33-40, 1996. http://dx.doi. org/10.1109/17.491266

CALABRESE, G. R\&D globalization in the car industry. International Journal of Automotive Technology and Management, v. 1, n. 1, p. 145-159, 2001. http://dx.doi. org/10.1504/1JATM.2001.000032

CAMUFFO, A. Rolling out a world car: globalization, outsourcing and modularity in the auto industry. Venice: Department of Business Economics and Management Ca' Foscari University of Venice, 2001. Disponivel em: <www.imvp.mit.edu/papers/0001/camuffo1.pdf>. Acesso em: 02 maio 2009.

CAUCHICK MIGUEL, P. A.; HSUAN, J. An exploratory investigation on modularity adoption in design and production through a case-based research in a Brazilian automaker. Product: Management \& Development, v. 8, n. 2, p. 173-181, 2010.

CHIESA, V. Globalizing R\&D around centres of excellence. Long Range Planning, v. 28, n. 6, p. 19-28, 1995. http:// dx.doi.org/10.1016/0024-6301(95)00048-N

CHIESA, V. Human resource management issues in global R\&D organizations: a case study. Journal of Engineering and Technology Management, v. 13, n. 1, p. 189-202, 1996. http://dx.doi.org/10.1016/S0923-4748(96)01003-X

CHIESA, V. Technology development control styles in multinational corporations: a case study. Journal of Engineering and Technology Management, v. 16, n. 1, p. 191-206, 1999. http://dx.doi.org/10.1016/S09234748(99)00005-3

CHIESA, V. Global R\&D Project Management and Organization: a Taxonomy. Journal of Product Innovation Management, v. 17, n. 1, p. 341-359, 2000. http://dx.doi.org/10.1016/S0737-6782(00)00049-7

CLARK, K. B.; WHEELWRIGHT, S. C. Revolutionizing product development: quantum leaps in speed, efficiency and quality. New York: The Free Press, 1992.

CLARK, K. B.; WHEELWRIGHT, S. C. Managing New Product and Process Development. New York: The Free Press, 1993.

CONSONI, F. Da tropicalização ao projeto de veículos: um estudo das competências em desenvolvimento de produtos nas montadoras de automóveis no Brasil. 2004. Tese (Doutorado)-Instituto de Geociências, Universidade Estadual de Campinas, Campinas, 2004.

COOPER, R. G. Winning at new products: accelerating the process from idea to launch. Reading: Perseus Books, 1993.

DIAS, A. V. C. Produto mundial, engenharia brasileira: integração de subsidiárias no desenvolvimento de produtos globais na indústria automobilística. 2003. Tese (Doutorado)-Universidade de São Paulo, São Paulo, 2003.

DIAS, A. V. C.; GALINA, S. V. R. Global product development: some case studies in the brazilian automotive and telecommunication industries. In: INTERNATIONAL CONFERENCE ON TECHNOLOGY POLICY AND INNOVATION, 4., 2000, Curitiba. Proceedings... Curitiba: Pontifícia Universidade Católica do Paraná, 2000.

DIAS, A. V. C.; SALERNO, M. S. Descentralização das atividades de pesquisa, desenvolvimento e engenharia de empresas transnacionais: uma investigação a partir da perspectiva de subsidiárias automotivas. Gestão \&
Produção, v. 16, n. 2, p. 187-199, 2009. http://dx.doi. org/10.1590/S0104-530X2009000200003

FLEURY, A.; FLEURY, M. T. Estratégias empresariais e formação de competências: um quebra cabeça caleidoscópico na indústria brasileira. São Paulo: Atlas, 2000.

FRANSMAN, M. Evolution of the telecommunications industry into the internet age. 2001. Disponivel em: <www.telecomvisions.com/articles/pdf/ fransmantelecomshistory.pdf>. Acesso em: 10 nov. 2008.

GALINA, S. V. R.; PLONSKI, G. A. Desenvolvimento global de produtos no setor de telecomunicações: uma taxonomia para a participação brasileira. In: CONGRESSO BRASILEIRO DE GESTÃO DE DESENVOLVIMENTO DE PRODUTOS, 2., 2000, São Carlos. Anais... São Carlos, 2000.

GALINA, S. V. R. Desenvolvimento global de produtos: o papel das subsidiárias brasileiras de fornecedores de equipamentos do setor de telecomunicações. 2003. Tese (Doutorado)-Universidade de São Paulo, São Paulo, 2003.

HARVEY, M. G.; GRIFFITH, D. A. The role of globalization, time acceleration, and virtual global teams in fostering successful global product launches. Journal of Product Innovation Management, v. 24, n. 1, p. 486-501, 2007. http://dx.doi.org/10.1111/j.1540-5885.2007.00265.x

HÖLTTA, K.; TANG, V.; SEERING, W. P. Modularizing product architectures using dendrograms. Cambridge: Massachusetts Institute of Technology, Center for Innovation in Product Development, 2003.

HUANG, C. C.; KUSIAK, A. Modularity in design of products and systems. IEEE Transactions on Systems, Man and Cybernetics, v. 28, n. 1, p. 66-77, 1998. http://dx.doi. org/10.1109/3468.650323

HUANG, C. C. Overview of modular product development. Proceedings of the National Science Council, Republic of China, Part A, v. 24, n. 3, p. 149-165, 2000.

HUMPHREY, J.; LECLER, Y.; SALERNO, M. S. Global Strategies and Local Local Realities: the Autoindustry in Emerging Markets. London: Macmillan Press Ltd., 2001.

INTERNATIONAL ORGANIZATION OF MOTOR VEHICLE MANUFACTURES - OICA. Disponével em: <http://oica. net/category/production-statistics $>$. Acesso em: 20 dez. 2008.

MIKKOLA, J. H. Modularity and interface management: the case of Schindler Elevators. Copenhagen Business School, Department of Industrial Economics and Strategy, 2001. Disponível em: <http://web.cbs.dk/departments/ivs/wp/ wp01-06.pdf>. Acesso em: 10 abr. 2009.

MILLER, R. Global ReD networks and large-scale innovations: the case of the automobile industry. Research Policy, v. 23, n. 1. p. 27-46, 1994. http://dx.doi.org/10.1016/00487333(94)90025-6

MUDAMBl, R.; MUDAMBI, S. M.; NAVARRA, P. Global Innovation in MNCs: The effects of subsidiary selfdetermination and teamwork. Journal of Product and Innovation Management, v. 24, n. 1, p. 442-455, 2007. http://dx.doi.org/10.1111/j.1540-5885.2007.00262.x

MUFFATTO, M. Platform strategies in international new product development. International Journal of Operations e Production Management,v. 19, n. 5-6, p. 449-459, 1999. http://dx.doi.org/10.1108/01443579910260766

OPPAT, K. Disseminative Capabilities: a case study of collaborative product development in the automotive industry. Schrifften zum europäischen Management, Gabler Edition Wissenschaft, 2008.

PANDREMENOS, J. et al. Modularity concepts for the automotive industry: a critical review. CIRP Journal of 
Manufacturing Science and Technology, v. 1, n. 1, p. 148152, 2009. http://dx.doi.org/10.1016/j.cirpj.2008.09.012

QUINTELLA, H. L. M. M.; ROCHA, H. M. Nível de maturidade e comparação dos PDPs de produtos automotivos. Revista Produção, v. 17, n. 1, p. 199-217, 2007. http://dx.doi. org/10.1590/S0103-65132007000100014

REDDY, A. S. P.; SIGURDSON, J. Emerging patterns of globalization of corporate $R \& D$ and scope for innovation capability building in developing countries? Science and Public Policy, v. 21, n. 1, p. 283-294, 1994.

REDDY, P. New trends in globalization of corporate R\&D and implications for innovation capability in host countries: a survey from India. World Development, v. 25, n. 11, p. 1821-1837, 1997. http://dx.doi.org/10.1016/S0305750X(97)00079-X

RONSTADT, R. Research and development abroad by US multinationals. Nova York: Praeger Publishers, 1977.

ROZENFELD, H. Modelo de Referência para o Desenvolvimento Integrado de Produtos. In: ENCONTRO NACIONAL DE ENGENHARIA DE PRODUÇÃO, 1997, Gramado. Anais... Gramado, 1997.

ROZENFELD, H. et al. Gestão de Desenvolvimento de Produtos: Uma referência para a melhoria do processo. São Paulo: Saraiva, 2006.

SALERNO, M. S. Nova configuração da cadeia automotiva brasileira. São Paulo: Departamento de Engenharia de Produção, Universidade de São Paulo, 2002. Relatório de Pesquisa.

SALERNO, M. S.; CAMARG0, 0. S.; LEMOS, M. B. Modularity ten years after: an evaluation of the Brazilian experience. International Journal of Automotive Technology and Management, v. 8, n. 4, p. 373-381, 2008. http://dx.doi. org/10.1504/1JATM.2008.020309

SALERNO, M. S. et al. A Nova Configuração da Cadeia Automotiva no Brasil: Rumo a um Pólo de Excelência em Projeto e Produção? 1. Fase levantamento extensivo junto às empresas de autopeças. USP, 2001. Disponível em: <www.poli.usp.br/pro/cadeia-automotiva>. Acesso em: 01 maio 2009.

SILVA, S. L. et al. Product development process in the Brazilian autoparts industry: management practices and trends. International Journal of Automotive Technology and Management, v. 4, p. 40-61, 2004. http://dx.doi. org/10.1504/1JATM.2004.004395

SÖDERQUIST, K. E. Organizing knowledge management and dissemination in new product development: lessons from 12 global corporations. Long Range Planning, v. 39, n. 1, p. 497-523, 2006. http://dx.doi.org/10.1016/j. Irp.2005.07.004

SUBRAMANIAM, M. Integrating cross-border knowledge for transnational new product development. Journal of Product Innovation Management, v. 23, n. 1, p. 541-555, 2006. http://dx.doi.org/10.1111/j.15405885.2006.00223.x

TOLED0, J. C. et al. Participation of suppliers in product development process: the case of the Fiat New Palio. Product: Management \& Development, v. 2, p. 5369, 2003.

TOLEDO, J. C. et al. Práticas de gestão no desenvolvimento de produtos em empresas de autopeças. Produção, v. 18 p. 405-422, 2008. http://dx.doi.org/10.1590/S010365132008000200015

ULRICH, K.; EPPINGER, S. D. Product design and development New York: McGraw-Hill, 1995.

VOSS, C. et al. Case Research in Operations Management. International Journal of Operations and Production Management, v. 22, n. 2, p. 195-219, 2002. http:// dx.doi.org/10.1108/01443570210414329

WALLACE, T. Innovation and Hybridization: Managing the introduction of lean production into Volvo do Brazil. International Journal of Operations and Production Management, v. 24, n. 8, p. 801-819, 2004. http:// dx.doi.org/10.1108/01443570410548239

YIN, R. G. Case study research - design and methods. London: Stage, 2001.

\section{Analysis of decentralization of new product development: an investigation in a company from the commercial vehicles sector Abstract}

The current literature identifies a trend of decentralization on new product development (NPD) at global level. In this context, this paper aims at analyzing the decentralization of global product development (GPD) in an assembler from the commercial vehicles industrial sector. The factors that contribute to decentralization of GPD are considered as variables in this investigation, as well as their causes. Given the nature of data (qualitative), context relevance (automotive sector), and research purpose, base-based research was adopted as the methodological approach. The study was conducted in an assembler in Brazil and in its headquarter in Germany. The results indicate that the organization reacts similarly to other economic industrial sectors (e.g. telecommunications) when facing the decision of decentralizing GPD. The interest of the local market and historical aspects of the evolution of the subsidiary were found to be relevant. It was also identified that modular design influences the process of decentralizing, contradictory to other studies. From these results, a proposal associated to the type of structure for GPD and adoption of modular design is made.

\section{Keywords}

Automotive industry. Decentralization of product development. Modularity. 